\title{
Gamma Rays and Neutrinos from SNR RX J1713.7-3946
}

\author{
G. Morlino ${ }^{\mathrm{a}}$, P. Blasi ${ }^{\mathrm{a}, \mathrm{b}}$, E. Amato ${ }^{\mathrm{a}}$ \\ ${ }^{a}$ INAF/Osservatorio Astrofisico di Arcetri, Largo E. Fermi, 5,50125 Firenze, Italy \\ ${ }^{b}$ INFN, Laboratori Nazionali del Gran Sasso, I-67010 Assergi (AQ), Italy
}

\begin{abstract}
The supernova paradigm for the origin of galactic cosmic rays can be tested using multifrequency observations of both non-thermal and thermal emission from supernova remnants. The smoking gun of hadronic acceleration in these sources can, however, only be provided by the detection of a high energy neutrino signal. Here we apply the theory of non-linear particle acceleration at supernova shocks to the case of the supernova remnant RX J1713.7-3946, which is becoming the stereotypical example of a possible hadronic accelerator after the detection of high energy gamma rays by the HESS telescope. Our aim is twofold: on one hand we want to address the uncertainties in the discrimination between a hadronic and a leptonic interpretation of the gamma ray emission, mainly related to the possibility of a statistical uncertainty in the energy determination of the gamma ray photons in the $\mathrm{TeV}$ region. On the other we want to stress how a $\mathrm{km}^{3}$ neutrino telescope would break the degeneracy and provide evidence for efficient cosmic ray acceleration in RX J1713.7-3946. A $3 \sigma$ evidence would require about two years of observation.
\end{abstract}

Key words: acceleration of particles - neutrinos - object: RX J1713.7-3946

\section{Introduction}

Supernova remnants (SNRs) are the most plausible sources of galactic cosmic rays. The recent detection of multi-TeV gamma radiation from several SNRs makes the case stronger, especially when coupled with multifrequency observations of the same remnants. However, despite all this progress, the evidence that SNRs are indeed the main contributors to Galactic cosmic rays remains circumstantial. A smoking gun evidence of efficient acceleration of cosmic rays in these sources can only come from the detection of high energy neutrinos, resulting from the decays of charged pions within the source.

The acceleration of cosmic rays at supernova blast waves is well described by the non linear theory of diffusive shock acceleration (NLDSA) (see [28] for a review).

Email addresses: morlino@arcetri.astro.it (G. Morlino), blasi@arcetri.astro.it (P. Blasi), amato@arcetri.astro.it (E. Amato)
This theory allows us to calculate the spectrum and spatial distribution of cosmic rays accelerated at a supernova shock taking into account the dynamical reaction of the accelerated particles on the shock and, in its most recent version [6, 16, 17], also the generation of magnetic field through streaming instability induced by the accelerated particles [13, 12] and the dynamical reaction of the amplified magnetic field on the plasma. Such a dramatic improvement in the quality of our theoretical approach allows us to finally compare theory with observations and make testable predictions for future observations.

One of the most important recent breakthroughs in establishing the SNR paradigm for the origin of cosmic rays has been the detection of narrow filaments of non-thermal $\mathrm{X}$-ray emission in the direction of supernova remnant rims $[10,9,26,25,40]$. The filaments are most commonly interpreted as the result of severe synchrotron energy losses of ultra relativistic electrons which are forced to radiate a large fraction of their energy (in the form of X-rays) in a narrow region downstream of the shock. The required 
strengths of the magnetic field are of order $\sim 100-500 \mu G$ downstream of the shock. Such large levels of magnetization might be the manifestation of magnetic field amplification in the upstream region, as due to the streaming of cosmic rays $[35,13,6]$. Not only the detection of large magnetic fields signals for efficient cosmic ray acceleration, but in turn large magnetic fields are needed for increasing the maximum energy of accelerated particles to the knee region [14], namely for efficient acceleration. Efficient cosmic ray acceleration and magnetic field amplification are two sides of the same coin.

On the other hand, it has been proposed that the interpretation of the narrow X-ray rims may be flawed [32]: they could in fact result from damping of magnetic field in the downstream region. The emission region would be narrow because the field disappears, not because particles lose energy effectively. The situation is currently subject of active debate: the damping would naively result in the appearance of filaments not only in X-rays, but also in the radio emission, and at present there is no evidence for such a phenomenon. In addition, the absence of magnetic field amplification (or a mitigation of the effect) would reduce the maximum energy achieved by the accelerated particles, unless the shock configuration is quasiperpendicular [23]. An additional possibility has been proposed in [20]: the magnetic field could be amplified downstream (and not upstream) because of the development of corrugations on the shock surface which result in eddies in which the magnetic field winds up and gets amplified. In order for this scenario to lead to large maximum momentum of the accelerated particles, the field direction must be very inclined with respect to the shock normal in the upstream region.

A powerful diagnostic tool for particle acceleration in SNRs is represented by the gamma ray emission in the $\mathrm{GeV}-\mathrm{TeV}$ energy region. The spectrum of the gamma ray emission, its extension to high energies and the shape of the cutoff all provide precious information on whether the radiation is of leptonic or hadronic origin. In [30] we discussed in detail the application of NLDSA to the case of the supernova remnant RX J1713.7-3946. If the HESS data on this remnant are taken at face value, then it is problematic to fit them in the context of a leptonic model for at least two reasons: 1) the spectral cutoff expected in leptonic scenarios leads to a gamma ray emission which falls short of the highest energy data points measured by the HESS telescope; 2) in any case, fitting both the TeV and X-ray data requires the presence of a very large background of infrared photons in the remnant.

The second point raised above should be considered as a circumstantial evidence against a leptonic model, but by itself it does not suffice to rule out this class of models. In principle the first point is rather solid, but experimental effects might weaken its significance: a statistical (and systematic) uncertainty in the energy determination affects the steep gamma ray spectrum by making it look smoother than it actually is so that convolving the theoretical prediction with an estimate of the uncertainty in the energy determination makes the leptonic spectrum look more similar to the hadronic case. We address this issue in a quantitative way here.

The hadronic interpretation appears to be more sound, but there are shortcomings in this case as well: first, the thermal X-ray flux expected from RX J1713.7-3946 is larger than the observed radiation of synchrotron origin. Second, the number of electrons needed to explain the observations is about $10^{-4}$ of the number density of accelerated protons, at odds with the $\mathrm{e} / \mathrm{p}$ ratio observed at Earth at energy $\sim 1-10 \mathrm{GeV}$.

Both these points are however rather weak at the present time. The first is based on the assumption that electrons and protons share the same temperature downstream. This condition is hardly achievable and in fact one can easily argue that the temperature of electrons should be much smaller than that of protons. On the other hand, Coulomb scattering might be sufficient to raise the electron temperature to a level large enough to excite emission lines of heavy elements [31]. The second point is equally weak in that electrons might be accelerated at different stages of the SNR. Moreover, recent data from PAMELA [1] and ATIC [18] suggest that a substantial contribution to the observed electron spectrum at Earth might come from sources other than SNRs [22, 37].

The safest way of proving or rejecting acceleration of hadrons in RX J1713.7-3946, as well as in other remnants, is to search for neutrinos produced in the decays of charged pions. In this paper we apply the NLDSA model developed by [5, 6], and previously used to describe the multifrequency spectrum of RX J1713.7-3946 [30], in order to calculate the expected neutrino flux from this remnant.

Previous attempts at estimating the neutrino flux from 
SNRs have been typically based on phenomenological approaches, building on the assumption of a power law approximation for the gamma ray spectrum and simple scaling relations between the gamma ray and neutrino spectra. In [8] the authors estimated a flux of 40 neutrino induced muons (and antimuons) per $\mathrm{km}^{2}$ per year from RX J1713.7-3946, using a power law for the $\gamma$-ray spectrum $f_{\gamma}(E) \propto E^{-2}$, based on CANGAROO observations; the maximum neutrino energy was assumed to be $\sim 10$ TeV. Neutrino oscillations, absorption and location of the detector were not taken into account. Such effects were included by [19], where $f_{\gamma}(E) \propto E^{-2.2}$ was used, the power law index being inferred from HESS data (no cut-off energy was assumed in this approach). The authors obtain a flux of neutrino induced muons $N_{\mu+\bar{\mu}} \simeq$ $10 \mathrm{~km}^{-2} \mathrm{yr}^{-1}$. Again based on HESS data, [41] used two different parametrizations for the $\gamma$-ray spectra, a power law plus an exponential cutoff at $E_{\gamma, \max }=12 \mathrm{TeV}$ and a broken power law (with a knee at $6.7 \mathrm{TeV}$ ), leading to $N_{\mu+\bar{\mu}}=4.8$ and $5.4 \mathrm{~km}^{-2} \mathrm{yr}^{-1}$ respectively. An attempt to extract the proton spectrum from the HESS data on the gamma ray flux (assumed to be of hadronic origin), and compute the neutrino flux based on that, was done in [39].

In the present paper we carry out the calculations by using our model of NLDSA which provides a self-consistent description of the acceleration of cosmic rays in the remnant. This leads to a shape of the gamma ray spectrum which is not a simple power law, due to the non linear effects induced by the dynamical reaction of the accelerated particles and the amplified magnetic field. At the same time we also obtain self-consistently the neutrino spectrum, and we use it to derive the expected number of events in a $\mathrm{km}^{3}$ neutrino telescope.

The paper is organized as follows: in $\S 2$ we summarize the technical aspects of NLDSA and its application to particle acceleration in supernova remnants. We also describe the calculations of the non thermal radiation from RX J1713.7-3946, with special attention for the gamma ray emission in both the hadronic and leptonic scenario. We discuss in detail the possibility to use present and future observations to discriminate between the two, once a statistical uncertainty in the energy determination of the photon events is taken into account. We show that a leptonic scenario convolved with a gaussian distribution of the photon energies with $\delta E / E \sim 30 \%$ or larger leads to the impossibility to distinguish the leptonic prediction from the hadronic one, at least using the HESS data on RX J1713.7-3946. The implications for future gamma ray telescopes are also briefly discussed. On these premises it is very important to aim at the detection of the associated neutrino signal, whose intensity is calculated in $\S 3$, where we also estimate the neutrino induced muon signal in a $\mathrm{km}^{3}$ neutrino detector. We conclude in $\$ 4$

\section{NLDSA and the non thermal emission of RX J1713.7-3946}

\subsection{Spectrum of accelerated protons and electrons}

In NLDSA theory, the overall shock structure and the outcome of the particle acceleration process are inextricably linked. When acceleration is efficient, the pressure of accelerated particles affects the shock dynamics, leading to the formation of a precursor, namely a region where the fluid velocity progressively decreases while approaching the shock from far upstream. At the same time the streaming of accelerated particles is responsible for the instabilities that lead to magnetic field amplification. In turn, the fluid profile in the precursor and the amplified, turbulent magnetic field, with the scattering it provides (and possibly the induced energy losses), determine the efficiency of particle acceleration and the resulting spectrum, including its high energy cutoff.

The shock structure and the accelerated particle spectrum are computed as in [30]: the basic structure of the calculation is the same proposed in [5] and [6], but with a crucial new aspect taken into account, namely the dynamical reaction of the self-generated magnetic field, which is included following the treatment of [16] and [17]. This means that the conservation equations at the shock and in the precursor are modified so as to include the magnetic contribution. The compression factor at the subshock, $R_{s u b}$, and the total compression factor, $R_{t o t}$, are deeply affected by this change, resulting in a decrease of the compression ratio in the precursor, $R_{t o t} / R_{s u b}$, as soon as the amplified magnetic field contributes a pressure comparable to that of the thermal gas upstream. This smoothening of the precursor reflects in spectra of accelerated particles which are closer to power laws, though the concavity typical of NLDSA remains visible [17].

The normalization of the proton spectrum is an output of our non linear calculation, once a recipe for injection has been established. Following [15], particles are 
injected immediately downstream of the subshock. The fraction of particles crossing the shock surface which are injected in the acceleration process, $\eta_{\text {inj }}$, can be written as

$$
\eta_{\text {inj }}=4 /(3 \sqrt{\pi})\left(R_{\text {sub }}-1\right) \xi^{3} e^{-\xi^{2}} \text {. }
$$

Here $\xi \sim 2-4$ is defined by the relation $p_{\mathrm{inj}}=\xi p_{\mathrm{th}, 2}$, where $p_{\mathrm{th}, 2}$ is the momentum of thermal particles downstream. $\xi$ parametrizes the poorly known microphysics of the injection process, but $p_{\mathrm{th}, 2}$ is an output of the problem: as a result, the injection efficiency is affected by the dynamical reaction exerted by the accelerated particles and by the amplified magnetic field.

Finally, the maximum momentum of the accelerated particles is determined following [14] for the computation of the acceleration time in the presence of a precursor.

For protons we use as a prescription the equality between the acceleration time and the age of the SNR:

$$
t_{a c c}\left(p_{p, \max }\right)=t_{S N R}
$$

For electrons, energy losses can be important. Their maximum momentum $p_{e \text {, max }}$ is determined by equating the acceleration time with the minimum between the time for energy losses and the age of the remnant. The loss time of electrons over a cycle of shock crossing needs to be weighed by the residence times, $t_{r}$, upstream and downstream, so that the condition for the maximum momentum, in the loss dominated case, can be written as:

$$
t_{a c c}(p)=\frac{t_{r, 1}(p)+t_{r, 2}(p)}{\frac{t_{r, 1}(p)}{\tau_{l, 1}\left(B_{1}, p\right)}+\frac{t_{r, 2}(p)}{\tau_{l, 2}\left(B_{2}, p\right)}}
$$

where $\tau_{l}$ denotes the loss time, and the indexes " 1 " and " 2 " refer to quantities measured upstream and downstream respectively. The residence times in the context of the non linear theory of particle acceleration can be written explicitely (from Eqs. (25) and (26) of [14]). Eq. (3) must be solved numerically for $p_{e, \max }$, contrary to the case of acceleration in the test particle regime. However an approximate analytical solution, valid when only synchrotron losses are important, was also proposed in [30].

As to the electron spectrum at the shock, $f_{e, 0}(p)$, this is easy to calculate for $p \ll p_{e, \max }$. In fact, at a given momentum $p$, the slope of the electron and proton spectrum is the same, if one assumes that both species experience the same diffusion coefficient. What is unconstrained $a$ priori is the relative normalization of the two spectra, $K_{e p}$, which can only be obtained by fitting the observations. This is reasonable since electrons do not exert any appreciable dynamical reaction on the shock.

The spectrum of electrons at energies around and above $p_{e, \max }$, namely the shape of the cutoff is harder to calculate in the context of non-linear theory. Since the spectra we find for electrons at $p<p_{e, \max }$ are not far from being power laws with slope $\sim 4$, we adopt the modification factor calculated by [44] for strong shocks in test particle regime. The resulting electron spectrum at the shock, in the loss dominated case, is:

$$
f_{e, 0}(p)=K_{e p} f_{p, 0}(p)\left[1+0.523\left(p / p_{e, \max }\right)^{\frac{9}{4}}\right]^{2} e^{-p^{2} / p_{e, \max }^{2}} .
$$

What is important to notice in this expression is that the cutoff is not a simple exponential, a fact which reflects in the shape of the synchrotron spectrum radiated by the electrons, making it different from what assumed by most of the previous work on the subject. On the other hand, if the maximum momentum is indeed determined by the age of the remnant, then the cutoff shape is expected to be exponential.

\subsection{Magnetic field amplification and compression}

The turbulent magnetic field close to the shock can be enhanced by several physical processes. However, here, as in [30], we focus on the amplification due to resonantly excited streaming instability induced by cosmic rays accelerated at the shock. Resonant streaming instability [35] is likely responsible for most of the magnetic field amplification in SNRs after the beginning of the Sedov phase [7], while the non-resonant mode of the same instability [12] is more effective at earlier times. When the predictions of linear theory are extrapolated to the non-linear regime of field amplification (which one is forced to do for lack of a better treatment), the resulting field strengths are in agreement with the values inferred by identifying the thickness of the X-ray filaments with the synchrotron loss length of the highest energy electrons. The strength of the magnetic field at the position $x$ upstream, $\delta B(x)$, in the absence of damping, can be estimated from the saturation condition, that, for modified shocks, reads [17]:

$$
p_{w}(x)=U(x)^{-3 / 2}\left[\frac{1-U(x)^{2}}{4 M_{A, 0}}\right],
$$


where $p_{w}(x)=\delta B(x)^{2} /\left(8 \pi \rho_{0} u_{0}^{2}\right)$ is the magnetic pressure normalized to the incoming momentum flux at upstream infinity, $U(x)=u(x) / u_{0}$ and $M_{A, 0}=u_{0} / v_{A}$ with $v_{A}$ the Alfvén velocity at upstream infinity, where only the background magnetic field, $B_{0}$, assumed parallel to the shock normal, is present. Eq. (5) correctly describes the effect of compression in the shock precursor through the term $U(x)^{-3 / 2}$. For the upstream temperature that we adopt in RX J1713.7-3946 (see below), damping in the upstream region is expected to be negligible.

The magnetic field downstream of the subshock is further enhanced by compression, according to:

$$
B_{2}=R_{\text {sub }} B_{1},
$$

where $B_{1}$ is the magnetic field immediately upstream of the subshock and we have used the fact that we are dealing with Alfvén waves, and hence turbulence perpendicular to the shock normal.

\subsection{Computation of the radiation fluxes and spectra}

The flux of non-thermal radiation at $\gamma$-ray photon energies is computed as in [30]. In that work we considered both possible scenarios for the origin of high energy photons in RX J1713.7-3946, namely a leptonic origin, through inverse Compton scattering (ICS) of ambient low energy photons by accelerated electrons, or a hadronic origin, from $\pi^{0}$ decay following nuclear collisions of relativistic protons.

In the hadronic scenario, the $\gamma$-ray flux from RX J1713.7-3946 is accompanied by a flux of neutrinos coming from the decay of charged pions that are produced in nuclear collisions together with neutral pions. We compute the fluxes and spectra in both channels using the approximated expressions for the cross sections as provided in Ref. [24].

The $\gamma$-ray and the neutrino fluxes, $\Phi_{i}^{0}\left(E_{i}\right)(i=\gamma, v)$, produced by $p$ - $p$ collisions from a source located at distance $d$ from Earth, can be expressed as follows:

$$
\Phi_{i}^{0}\left(E_{i}\right)=\frac{c}{4 \pi d^{2}} \int d \mathbf{r} n(\mathbf{r}) \int_{E_{i}}^{\infty} d E_{p} f_{p}\left(\mathbf{r}, E_{p}\right) \frac{d \sigma_{i}\left(E_{p}, E_{i}\right)}{d E_{i}} .
$$

In this expression, the apex 0 is used to indicate the neutrino flux that would have been seen at the Earth in the absence of neutrino oscillations. For gamma rays the two fluxes clearly coincide. Here $n$ is the gas (target) density in the SNR and $f_{p}\left(\mathbf{r}, E_{p}\right)$ is the distribution function of accelerated protons at the location $\mathbf{r}$ at a given energy $E_{p}$. In general both quantities depend on the location in the shell, but here we assume them to be constant in the region enclosed between the contact discontinuity and the forward shock and vanishing outside this region. Finally, $d \sigma_{i} / d E_{i}$ is the inclusive differential cross section for the production of particles of type $i$. This quantity is usually expressed through the total inelastic $p$ - $p$ cross section, $\sigma_{\text {inel }}$, and the dimensionless distribution function $F_{i}\left(x_{i}, E_{p}\right)$ for secondaries, as

$$
\frac{d \sigma_{i}\left(E_{p}, E_{i}\right)}{d E_{i}}=\frac{\sigma_{\text {inel }}\left(E_{p}\right)}{E_{p}} F_{i}\left(x_{i}, E_{p}\right)
$$

where $x_{i} \equiv E_{i} / E_{p}$ is the fraction of proton energy transferred to the secondary particle. For the functions $F_{i}$, which effectively enclose all the details of the hadronic processes involved, we use the analytical approximation derived in [24] on the basis of numerical simulations of $p$ - $p$ collisions with the publicly available code SIBYLL. The analytical formulae provide a very good description of the flux and energy distribution of secondaries for energies above $0.1 \mathrm{TeV}$. As far as photons are concerned, $F_{\gamma}$ also includes the contribution of $\eta$ meson decay, in addition to that of $\pi^{0}$, with an overall accuracy of order a few $\%$. The estimate of neutrino fluxes is slightly less accurate, because $F_{v}$ only includes the decay of charged pions, while neglecting the contribution from $K$-mesons, and therefore leading to underestimate the neutrino flux by about $10 \%$. At energies lower than $100 \mathrm{GeV}$, and down to the rest energy of the $\pi$-meson, we use the extrapolated formulae provided again by [24], that should be accurate within $10 \%$.

The flux and spectrum of ICS photons is calculated by using the exact kernel for ICS, with the full Klein-Nishina (KN) cross section [33]. The main target photon field contributing to emission in the high energy $\gamma$-ray band is provided by dust-processed infrared photons, described by a blackbody spectrum with temperature $\sim 20 \mathrm{~K}[34]$. Following the instance of the IR+Optical photon background in the interstellar medium (ISM) we assume that the ratio of the optical to infrared energy densities remains $\sim 20$, while the energy density of IR light, $W_{I R}$, is left as a free parameter (in the ISM, $W_{I R} \approx 0.05 \mathrm{eV} \mathrm{cm}^{-3}$ ). 
In order to compute the spectra of accelerated particles and the resulting emission, we need estimates for a number of environmental parameters relative to RX J1713.73946 , namely its distance $(d)$, age $\left(t_{S N R}\right)$, expansion velocity $\left(u_{0}\right)$, and the values of temperature $\left(T_{0}\right)$, density $\left(n_{0}\right)$, and magnetic field strength $\left(B_{0}\right)$, in the surrounding medium. In addition to these, the only free parameters of the model are: $\xi$, entering Eq. (1), and $K_{e p}$, defining the ratio between accelerated electrons and protons. In the ICS scenario an additional free parameter is the above mentioned $W_{I R}$.

The uncertainties on the various parameters and how they affect the results of our calculations are thoroughly discussed in [30]. Here we only summarize the values that we have found to provide the best fit to the multifrequency data in both scenarios. Our adopted values of distance, age, expansion velocity and temperature are: $d=1 \mathrm{kpc}, t_{S N R}=1600 \mathrm{yr}$ (consistent with the historical chinese record of a supernova explosion in AD 393 [43]), $u_{0}=4300 \mathrm{~km} / \mathrm{s}, T_{0}=10^{6} \mathrm{~K}$. Other parameters are, in the hadronic scenario: $n_{0}=0.12 \mathrm{~cm}^{-3}, B_{0}=2.6 \mu G$, $\xi=3.8$; in the leptonic scenario, instead: $n_{0}=0.01 \mathrm{~cm}^{-3}$, $B_{0}=1.5 \mu G, \xi=4.1$.

The resulting values of magnetic field strengths, acceleration efficiency and maximum energy of the accelerated particles are quite different in the two cases: the hadronic scenario entails efficient acceleration, with a fraction of accelerated particles corresponding to about $10^{-4}$ and a maximum proton energy exceeding $10^{14} \mathrm{eV}$. In the leptonic case the fraction of accelerated protons drops to less than $10^{-5}$, corresponding to an energy conversion efficiency of about $2 \%$. The magnetic field downstream is of order $20 \mu G$, to be compared with $B_{2} \sim 100 \mu G$ for the first scenario (in agreement with the value inferred from the synchrotron loss length interpretation of the rim thickness). The ratio between the number density of accelerated electrons and protons at the shock turns out to be $K_{e p} \sim 10^{-4}$ if acceleration is efficient. On the other hand, inefficient acceleration, and the lower value of the magnetic field associated with the leptonic scenario, would favour $K_{e p} \sim 10^{-2}$, in agreement with measurements of the diffuse galactic cosmic rays. However, in order to fit both the X-ray and the gamma-ray fluxes in the context of this purely leptonic scenario a local energy density of infrared radiation $\sim 24$ times larger than the galactic average is required.

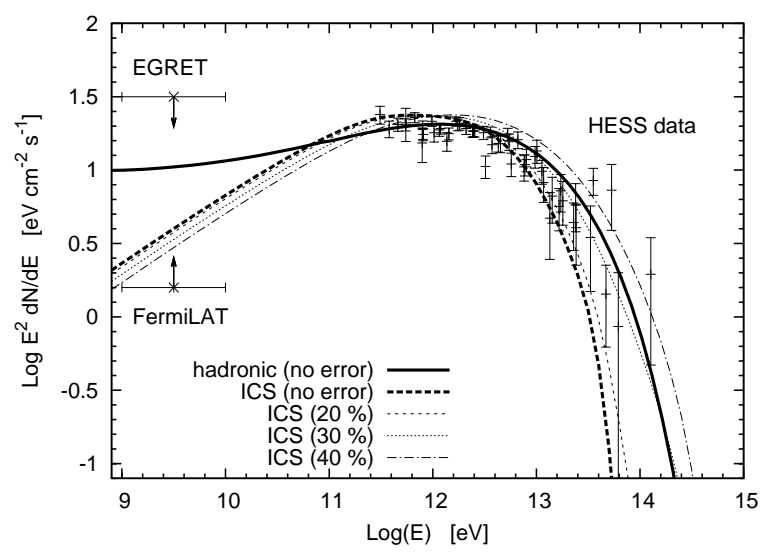

Figure 1: Hadronic versus leptonic scenarios for the origin of $\mathrm{TeV}$ emission from RX J1713.7-3946. In both cases, the flux is computed for the best-fit values of the parameters, as specified in the text. The thick solid line represents the spatially integrated spectral energy distribution of photons from $\pi^{0}$ decay in the hadronic scenario, while the thin solid line refers to ICS in the leptonic scenario. Symbols represent all available HESS data and also plotted are EGRET upper limit and FermiLAT sensitivity for $\mathrm{GeV}$ energy photons from this source. Other lines on the plot represent the result of convolution of the thin solid line with gaussians of different widths as explained in the text.

In Fig. 1 we plot the gamma ray flux as obtained through our calculations, for both a hadronic model (thick solid line) and a leptonic one (thick dashed line) (see [30]). Taken at face value, the curves in Fig. 1 clearly show that the hadronic scenario reproduces the high energy observations much better, in particular the highest energy data points of HESS. On the other hand, the possibility to discriminate between the two models relies upon the assumption that the statistical uncertainty in the energy determination is sufficiently small. In order to address this point, we calculate the theoretical prediction for the leptonic scenario in the case in which there is a statistical uncertainty in the energy determination $\sigma(E)=\chi E$ with $\chi=0.2,0.3,0.4$. Our results are shown again in Fig. 1 (different line-types are labelled in the figure).

The thin curves in the figure are obtained by convolving the predicted ICS flux at a given energy, $\phi_{\mathrm{ICS}}(E)$, with a gaussian of given width, namely :

$$
\Phi_{\mathrm{ICS}}^{\prime}(E)=\int d E^{\prime} \frac{\Phi_{\mathrm{ICS}}\left(E^{\prime}\right)}{\sqrt{2 \pi \sigma^{2}(E)}} \exp \left[-\frac{\left(E-E^{\prime}\right)^{2}}{2 \sigma^{2}(E)}\right] .
$$


The conclusion that these curves lead to is that a statistical uncertainty larger than $30 \%$ in the energy region above $10 \mathrm{TeV}$ would inhibit the discrimination between the two models, based on HESS data alone. The nominal energy resolution of HESS telescope is around 15\% [4], sufficient to allow for such discrimination. The main issue in this case is the data statistics: the main differences between the two scenarios, and the superior quality of the fit obtained within the hadronic one, show at the highest energy, where the statistical significance of the data points is not very high, as shown by the large error bars in Fig. 1 The role of the energy resolution is however important also in view of future gamma-ray telescopes, like CTA, whose design is currently being discussed by the scientific community.

In addition to all this, it is worth keeping in mind that the Fermi satellite is expected soon to provide another crucial bit of information to this debate, in that the level of detection (or the non detection) should clarify the issue of a leptonic or hadronic origin for the gamma ray emission from RX J1713.7-3946, independent of the shape of the high energy cutoff (see the low energy part of Fig.11).

\section{Neutrino signal}

The computation of the neutrino flux in the absence of neutrino oscillations was carried out in the assumption of perfect isospin symmetry $\left(\Phi_{\pi^{0}} \simeq \Phi_{\pi^{+}} \simeq \Phi_{\pi^{-}}\right)$, which leads to equal neutrino and antineutrino fluxes of a given flavor. For our purposes this is a good approximation.

The neutrino flux at the Earth is related to $\Phi_{v}^{0}$ through the oscillation probabilities:

$$
\Phi_{v_{l}}(E, d)=\sum_{l^{\prime}=e, \mu, \tau} P_{l l^{\prime}}(E, d) \Phi_{v_{l^{\prime}}}^{0}(E) .
$$

The transition probabilities, $P_{l l^{\prime}}$, are in general functions of energy and travel length, but since at the energies considered here the oscillation lengths are very short compared with the typical size of a SNR, the oscillation probability can be space averaged. The resulting flux of $v_{\mu}$ crossing the Earth, not taking into account absorption, is:

$$
\Phi_{v_{\mu}}=P_{\mu \mu} \Phi_{v_{\mu}}^{0}+P_{e \mu} \Phi_{v_{e}}^{0}=0.4 \Phi_{v_{\mu}}^{0}+0.2 \Phi_{v_{e}}^{0},
$$

with an identical equation holding for antineutrinos. The errors due to the uncertainties in the oscillation parameters are negligible $(\sim 5 \%)$. Since at the source $v_{\mu}$ and $v_{e}$ are produced in a ratio $\{2: 1\}$, the effect of oscillations translates into a flux of muon neutrinos at Earth that is $\sim 50 \%$ of that produced at the source: $\Phi_{v_{\mu}}=0.5 \Phi_{v_{\mu}}^{0}$.

The flux of neutrinos and antineutrinos of each flavor expected at Earth is shown in Fig. 2, and compared with the flux of gamma rays (solid line). The flux of neutrinos has to be compared with the background whose main contribution comes from atmospheric neutrinos. The shaded region shown in Fig. 2 refers to the theoretical prediction for the atmospheric neutrino flux as we explain below.

Following the estimates of [42, 21, 2] the atmospheric neutrino flux above $1 \mathrm{TeV}$ can be approximated as:

$$
\Phi_{v_{\mu}+\bar{v}_{\mu}}^{\mathrm{atm}}\left(E_{v}\right) \simeq 4.6 \times 10^{-8}\left(\frac{E_{v}}{1 \mathrm{TeV}}\right)^{-3.7} \mathrm{TeV}^{-1} \mathrm{~cm}^{-2} \mathrm{~s}^{-1} \mathrm{sr}^{-1}
$$

with an uncertainty of $\sim 40 \%$ due to the experimental error on the primary $\mathrm{CR}$ spectrum and composition and on theoretical models of hadronic interactions. Notice that the background flux also depends on zenith angle due to the different thickness of atmosphere to be crossed by cosmic rays coming from different directions. This dependence is included in the thickness of the shaded region by averaging on all possible neutrinos' incoming directions (see [11] for a detailed description of the mean uncertainties in the neutrino flux determination). Notice that for atmospheric neutrinos we neglect the contribution of oscillations, which is relevant only for energies $\lesssim 10 \mathrm{GeV}$ [27, §6.1].

With respect to Eq. (12) the atmospheric background shown in Fig. 2 is rescaled to a solid angle corresponding to a cone of semi-aperture 0.5 degrees. The motivation for choosing this value of the angle is twofold: it represents a reasonable estimate for the angular resolution of a neutrino telescope at these energies, and it also corresponds to approximately the angular size of the shell of RX J1713.7-3946. A smaller value of the aperture angle would imply a better chance to detect the signal than estimated below.

In the following we specialize our predictions to the case of a $\mathrm{km}^{3}$ neutrino telescope, for which the neutrino detection occurs by measuring the Cherenkov light from $v$-induced muons produced by charged-current interac- 


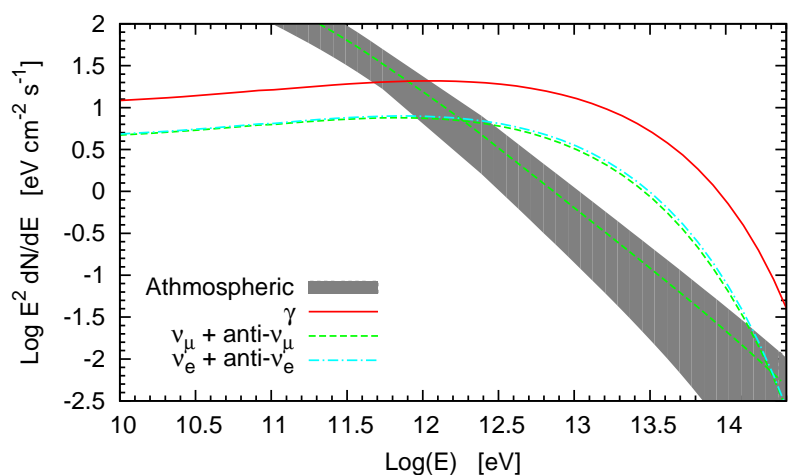

Figure 2: Fluxes of photons and neutrinos (plus anti-neutrinos) from RX J1713.7-3946 predicted in the hadronic scenario. Both electronic and muonic neutrinos contributions are shown. The shaded region represents the range of theoretical predictions for the atmospheric muonic neutrino (plus anti-neutrino) flux integrated in a cone of semi-aperture $0.5^{\circ}$.

tions of $v_{\mu}$ and $\bar{v}_{\mu}$ in the matter just below the detector. For the computation of the production rates of $\mu$ and $\bar{\mu}$, we follow the method of Ref. [19].

Since neutrinos can only be detected when the source is below the horizon of the detector, we introduce an average live-time of the source, in the form of a parameter $f_{\text {liv }}$, representing the fraction of time during which this condition is satisfied. For a neutrino telescope as ANTARES and RX J1713.7-3946 as the source, one can estimate $f_{\text {liv }} \approx 78 \%$ [19]. On the other hand, absorption of neutrinos while crossing the Earth leads to an energy dependent reduction of the detected flux. In fact the Earth becomes opaque to neutrinos at energies $E_{v} \approx 1 \mathrm{PeV}$ (when $\sigma$ becomes larger than $10^{-33} \mathrm{~cm}^{2}$ ). For neutrino energies of $\sim 100 \mathrm{TeV}(10 \mathrm{TeV})$ the signal is reduced by about $20 \%$ $(5 \%)$.

Taking into account these two effects, the number of muons with energy $E_{\mu}>E_{t h}$, crossing an area $A$ during the observation time $T$ can be written as:

$$
\begin{gathered}
N_{\mu}=f_{\text {liv }} A T \int_{E_{t h}}^{\infty} d E_{v} \Phi_{v}\left(E_{v}\right) Y_{\mu}\left(E_{v}, E_{t h}\right) \times \\
{\left[1-\bar{a}_{v}\left(E_{v}\right)\right],}
\end{gathered}
$$

with a similar equation holding for $N_{\bar{\mu}}$. In Eq. (13), $\bar{a}_{v}\left(E_{v}\right)$ is the mean coefficient for neutrino absorption through the Earth, including only charged-current interactions and

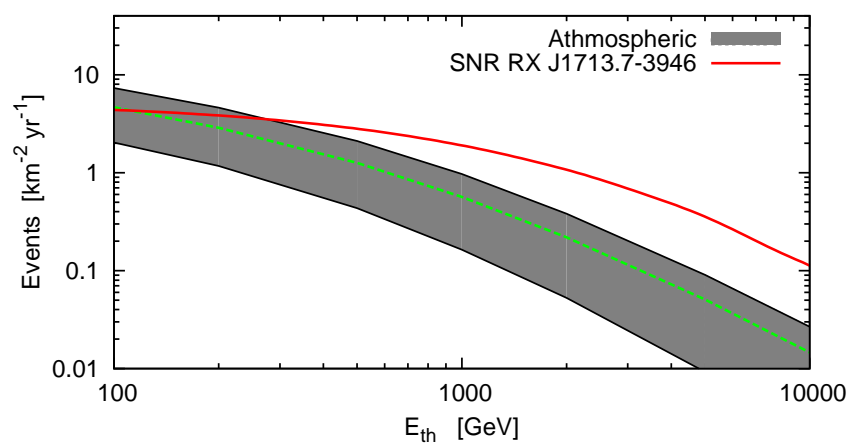

Figure 3: Neutrino event rate per year from RX J1713.7-3946 (solid line) as a function of muon energy threshold for a neutrino telescope with 1 $\mathrm{km}^{2}$ effective area. The dashed line shows the event rate expected from atmospheric neutrinos integrated in a cone of semi-aperture $0.5^{\circ}$.

computed for a fixed Earth thickness resulting from averaging over the observation time [19]. $Y_{\mu}\left(E_{v}, E_{t h}\right)$ is the muon yield, namely the probability that a neutrino with energy $E_{v}$ produces a muon with energy $E_{\mu}>E_{t h}$ that crosses the detector area. This is obtained by integration over the muon energy, $E_{\mu}$, of the neutrino interaction cross section multiplied by the muon range, $R\left(E_{\mu}, E_{t h}\right)$ :

$$
Y_{\mu}=\rho_{\mathrm{H}_{2} \mathrm{O}} N_{A} \int_{E_{t h}}^{E_{v}} d E_{\mu} \frac{d \sigma_{c c}}{d E_{\mu}}\left(E_{v}, E_{\mu}\right) R\left(E_{\mu}, E_{t h}\right) .
$$

Here $\rho_{\mathrm{H}_{2} \mathrm{O}}$ is the water density and $N_{A}$ is Avogadro's number. Both the muon range in water and the neutrino interaction probability are taken from [19]. The latter is calculated by using the deep inelastic scattering formula for the charged-current cross-section, $\sigma_{c c}$, and the distribution functions of partons as calculated by [29].

In Fig. 3 the solid line shows the resulting $N_{\mu}+N_{\bar{\mu}}$ for $A=1 \mathrm{~km}^{2}$ and $T=1 \mathrm{yr}$ as a function of the energy threshold. For $E_{t h}=50 \mathrm{GeV}$ the number of events per year is 4.7, compatible with the findings of [41]. The shaded region represents the muon background produced by atmospheric neutrinos, which can be computed as a function of the energy threshold, $E_{t h}$, by substituting Eq. (12) into Eq. (13). The result is normalized to an aperture angle of $0.5^{\circ}$, for the reasons explained above. The uncertainties represented by the shaded region have the same origin as for Fig. 2

The spectrum of neutrinos from RX J1713.7-3946 
Table 1: Comparison between the expected signal and the atmospheric neutrino background. The last column shows the observation time (in years) required to obtain a signal from RX J1713.7-3946 with a significance level of $3 \sigma$.

\begin{tabular}{cccc}
\hline$E_{t h}(\mathrm{GeV})$ & $N_{\mu+\bar{\mu}}$ & $N_{\mu+\bar{\mu}}^{\mathrm{atm}}$ & $\mathrm{yrs}(3 \sigma)$ \\
\hline \hline 100 & 4.4 & 4.7 & 2.16 \\
500 & 2.8 & 1.3 & 1.44 \\
1000 & 1.9 & 0.57 & 1.41 \\
\hline
\end{tabular}

dominates the atmospheric background at energies above $\sim 300 \mathrm{GeV}$. In Table 1 we report the time (in years) of observation needed to obtain a signal with significance level of $3 \sigma$, assuming a simple Poisson distribution of events and a unit detection efficiency. One can see that if RX J1713.7-3946 is indeed a hadronic accelerator a signal at the $3 \sigma$ level could be seen in about 2 years of observation at energies above $500 \mathrm{GeV}$.

\section{Conclusions}

In this paper we described the impact of non linear diffusive shock acceleration for the gamma ray and neutrino production in SNRs. The intricate connection between particle acceleration, magnetic field amplification, dynamical reaction of the particles and magnetic field, and the radiation produced by the accelerated particles (electrons and protons) in principle allow, if taken at face value, to impose strong constraints on the ability of SNRs to accelerate cosmic rays. An instance of how to use this powerful tool was provided in [30], where particle acceleration was described in terms of the non linear theory of Refs. [5, 6]. The magnetic field amplification was assumed to be due to resonant streaming instability and both the dynamical reaction of the accelerated particles and of the amplified field were taken into account. The basic conclusion reached in [30] is that the hadronic interpretation of the HESS data automatically leads to a downstream magnetic field which is in agreement with that inferred from X-ray observations if the rims of non-thermal emission are interpreted as the result of synchrotron losses. The non-thermal X-ray spectrum, as measured by Suzaku [38], was also reproduced with unprecedented accuracy. On the other hand, a satisfactory fit to the data within a leptonic scenario could only be achieved by considerably reducing the particle injection efficiency, through fine-tuning of the only free parameter of our calculations, $\xi$, as defined in $\$ 2.1$ Moreover, in order for the leptonic model to fit HESS data at all energies one is forced to require the presence of a diffuse background of infrared light exceeding that observed in the interstellar medium by more than a factor 20 . These requirements become less stringent if one decides not to include in the fit the highest energy HESS data points, which strongly constrain the maximum energy of the radiating electrons.

Since the discrimination between the two models, hadronic and leptonic, is based on such a tricky region from the observational point of view, we decided to address here the issue of how well the photon energies need to be reconstructed at the telescope in order to tell the difference between the predictions of a leptonic and a hadronic model. The issue is of particular importance since the different predictions are of relevance in the energy region where the particle spectra (and the gamma ray spectrum) are already sharply falling and a small statistical uncertainty in the energy determination may have sizable implications on the shape of the observed spectrum. Our calculations show that a statistical uncertainty in the energy determination larger than about $30 \%$ would inhibit the possibility to discriminate between a hadronic and a leptonic interpretation of $\mathrm{TeV}$ data. Of course a systematic uncertainty would strengthen the problem. These points need to be taken into account for the design of future telescopes, such as CTA. Needless to say that the observation of $\mathrm{TeV}$ emitting SNRs with the Fermi gamma ray telescope will definitely contribute to settle the debate. This should certainly be the case for RX J1713.7-3946 (see also [30]).

In the absence of clear multifrequency evidence however, the smoking gun that SNRs are efficient cosmic ray accelerators can only be provided by the unambiguous detection of neutrinos. Here we used the same non linear theory of particle acceleration to infer the number of neutrino induced muons in a $\mathrm{km}^{3}$ neutrino telescope. The flux is compared with the appropriate background of atmospheric neutrinos. At the distance of RX J1713.7-3946 and assuming a hadronic interpretation of HESS data, we predict that a $3 \sigma$ detection should be achieved by $\mathrm{km}^{3}$ neutrino telescope at the Antares' location (which is in 
the right hemisphere to detect RX J1713.7-3946) in about 2 years of observation.

\section{Acknowledgments}

We are grateful to Damiano Caprioli and Gamil Cassam-Chenai for continuous discussion and to Teresa Montaruli for reading the manuscript and providing useful comments. This work was partially supported by MIUR (under grant PRIN-2006) and by ASI through contract ASI-INAF I/088/06/0.

\section{References}

[1] Adriani, O., et al (PAMELA Collaboration), preprint arXiv0810.4995

[2] Agrawal, V., Gaisser, T. K., Lipari, P. \& Stanev, T. 1996, Phys. Rev. D, 53, 1314

[3] Aharonian, F., et al. (H.E.S.S. Collaboration), 2007, A\&A, 464, 235

[4] Aharonian, F., Buckley, J., Kifune, T. and Sinnis, G., 2008, Rep. Prog. Phys., 71, 096901

[5] Amato, E., and Blasi, P., 2005, MNRAS Lett., 364, 76

[6] Amato, E., Blasi, P., 2006, MNRAS, 371, 1251

[7] Amato, E., Blasi, P., 2008, MNRAS, 0, 0, Preprint arXiv:0806.1223

[8] Alvarez-Muñiz, J. and Halzen, F., 2002, ApJ, 576, L33

[9] Bamba, A., Yamazaki, R., Yoshida, T., Terasawa, T., Koyama, K., 2005, ApJ, 621, 793

[10] Bamba, A., Yamazaki, R., Ueno, M. \& Koyama, K., 2003, ApJ, 589, 827

[11] Barr, G. D., Robbins, S., Gaisser, T. K. and Stanev, T., 2006, Phys. Rev. D74, 094009

[12] Bell, A.R., 2004, MNRAS 353, 550

[13] Bell, A.R., 1978, MNRAS, 182, 147
[14] Blasi, P., Amato, E., Caprioli, D., 2007, MNRAS, 375,1471

[15] Blasi, P., Gabici, S., Vannoni, G., 2005, MNRAS, 361,907

[16] Caprioli, D., Blasi, P., Amato, E. \& Vietri, M., 2008a, ApJ Letters, 679, 139

[17] Caprioli, D., Blasi, P., Amato, E. \& Vietri, M., 2008b, MNRAS, submitted, arXiv:0807.4261

[18] Chang, J., et al. [ATIC Collaboration], 2008, Nature 456,362

[19] Costantini, M. L and Vissani, F., 2005, APh, 23, 466

[20] Giacalone, J., \& Jokipii, J. R. 2007, ApJ Letters, 663, L41

[21] Honda, M., Kajita, T., Kasahara, K., \& Midorikawa, S., 1995, Phys. Rev., D 52, 4985

[22] Hooper, D., Blasi, P., and Serpico, P., Preprint arXiv:0810.1527 (JCAP, in press)

[23] Jokipii, J. R. 1987, ApJ, 313, 842

[24] Kelner, S. R., Aharonian, F. A. and Bugayov, V. V., 2006, Phys. Rev., D 74, 034018

[25] Lazendic, J.S., Slane, P.O., Gaensler, B.M., Reynolds. S.P., Plucinsky, P.P., and Hughens, J.P. 2004, ApJ, 602, 271

[26] Lazendic J.S., Slane, P.O., Gaensler, B.M., Plucinsky, P.P., Hughes, J.P., Galloway \& D.K., Crawford, F., 2003, ApJ Lett, 593, 27

[27] Lipari, P., 2006, Nucl. Instrum. Meth., A567, 405

[28] Malkov, M.A., Drury, L.O'C, 2001, Rep. Progr. Phys., 64, 429

[29] Martin, A. D., Stirling, W. J., Thorne, R. S. and Watt, G., 2007, PhLB, 652, 292

[30] Morlino, G., Amato, E. and Blasi, P., 2008, MNRAS, accepted preprint (arXiv:0806.1223)

[31] Patnaude, D., Ellison, D., \& Slane, P., 2009, AAS meeting, 213, 359.01, preprint (arXiv:0902.2481v1) 
[32] Pohl, M., Yan, H. \& Lazarian, A., 2008, ApJ Lett, 626, 101

[33] Rybicki, G. B., Lightman, A. P., 1985, Radiative Processes In Astrophysics, 1st edn., WileyInterscience, Weinheim

[34] Schlegel, D. J., Finkbeiner, D. P. \& and Davis, M., 1998, ApJ, 500, 525

[35] Skilling, J., 1975a, MNRAS, 172, 557

[36] Skilling, J., 1975b, MNRAS, 173, 255

[37] Yuksel, H., Kistler, M.D., and Stanev, T., preprint arXiv:0810.2784

[38] Tanaka, T., et al., Preprint arXiv:0806.1490

[39] Villante, F.L. \& Vissani, F., 2007, Phys. Rev., D 76, 125019

[40] Vink, J. \& Laming. J.M., 2003, ApJ, 584, 758

[41] Vissani, F., 2006, APh., 26, 310

[42] Volkova, L. V., 1980, Sov. J. Nucl. Phys., 31, 784

[43] Wang, Z.R., Qu, Q.-Y., \& Chen, Y., 1997, A\&A, 318, L59

[44] Zirakashvili, V. N. \& Aharonian, F., 2007, A\&A, 465,695 